\title{
Hybrid high gradient permanent magnet quadrupole
}

\begin{abstract}
P. N'gotta, G. Le Bec, and J. Chavanne
European Synchrotron Radiation Facility, 71 Avenue des Martyrs, 38000 Grenoble, France

(Received 4 March 2016; revised manuscript received 15 November 2016; published 20 December 2016)

This paper presents an innovative compact permanent magnet quadrupole with a strong gradient for potential use in future light source lattices. Its magnetic structure includes simple mechanical parts, rectangular permanent magnet blocks and soft iron poles. It has a wide aperture in the horizontal plane to accommodate an x-ray beam port, a common constraint in storage ring-based light sources. This specificity introduces field quality deterioration because of the resulting truncation of the poles; a suitable field quality can be restored with an optimized pole shape. A $82 \mathrm{~T} / \mathrm{m}$ prototype with a bore radius of $12 \mathrm{~mm}$ and a $10 \mathrm{~mm}$ vertical gap between poles has been constructed and magnetically characterized. Gradient inhomogeneities better than $10^{-3}$ in the good field region were obtained after the installation of special shims.
\end{abstract}

DOI: 10.1103/PhysRevAccelBeams.19.122401

\section{INTRODUCTION}

Storage ring-based light sources undergo constant improvements in order to increase the brilliance and the coherence of the x-ray beams. Recently several facilities have launched upgrade projects based on multibend achromat lattices [1]. The European Synchrotron Radiation Facility-Extremely Brilliant Source (ESRF-EBS) upgrade is based on a 7-bend achromat lattice that contributes to a dramatic reduction of the horizontal emittance by a factor of 30 [2]. This lattice design requires strong quadrupoles with gradients ranging from 50 to $100 \mathrm{~T} / \mathrm{m}$. To reach such strengths, the magnet apertures are significantly reduced: from typically $70 \mathrm{~mm}$ down to $25 \mathrm{~mm}$ in the ESRF-EBS case. The reduction of magnet apertures makes the permanent magnet (PM) technology very attractive for some magnets provided that they are fixed field or with limited field tuning $( \pm 5)$. Indeed, introduction of PM structures may lead to better performances for a more compact design. Furthermore, with this technology running costs can be very low.

PM multipoles were introduced by Halbach [3]. This technology is extensively used for the construction of insertion devices. However, introduction of PM multipole devices in accelerator lattices has been very limited up to now. Some exceptions can be mentioned such as the use of low field PM magnets for the Fermilab recycler [4], and the development of PM multipoles and hybrid magnets for the final focusing of colliders [5-8]. It should be noted that a significant number of dipoles will be PM based in the new ESRF lattice [9-11]. In the case of quadrupoles, the PM technology needs further development (field tuning, field

Published by the American Physical Society under the terms of the Creative Commons Attribution 3.0 License. Further distribution of this work must maintain attribution to the author $(s)$ and the published article's title, journal citation, and DOI. stability with temperature variation, manufacturing process) before being installed in large scale facilities: this is the subject of the present paper. Compared to previous PM quadrupoles, this design combines high gradient and especially an improved field quality.

After a brief introduction on multipolar analysis, the design parameters for the magnets are presented and the performance criteria for magnet designs are defined (Secs. I and II).

The design and technological choices for a hybrid PM quadrupole, including iron poles, are presented in detail. A 3D fully parametrized model was built with RADIA, a magnetostatic computer code developed at the ESRF [12]. This model has been used for optimizing the magnet (Sec. III) and for analyzing its sensitivity to various errors (Sec. IV).

A PM quadrupole prototype was built and characterized. Section $\mathrm{V}$ presents the mechanical design of the magnet, its construction, the magnetic measurements, and finally the shimming method used for improving the field quality.

\section{MAGNET DESIGN CONSIDERATIONS}

\section{A. Strength and homogeneity}

The main magnetic specifications of a multipole magnet are the strength of its multipole components, denoted $Q$ in the following, and the spatial homogeneity of this component. In order to define these statements, let us introduce the complex magnetic field generated in the aperture of a multipolar structure:

$$
B(z)=B_{Y}+i B_{X}=\sum_{n=1}^{\infty} C_{n}\left(\frac{z}{R_{0}}\right)^{n-1},
$$

where $z=x+i y$ is the complex representation of any point in the aperture. $C_{n}=b_{n}+i a_{n}$ are the complex multipolar coefficients composed of normal coefficients 
$b_{n}$ and skew coefficients $a_{n}$. The unit of $C_{n}$ is Tesla. $R_{0}$ is the reference radius. The strength of an ideal multipolar structure of order $N$ is defined as

$$
Q_{N}\left[\mathrm{~T} / \mathrm{m}^{N-1}\right]=\frac{b_{N}}{R_{0}^{N-1}} .
$$

For a dipole $(N=1), Q_{1}$ corresponds to the magnetic induction $B$ in the gap. For a quadrupole $(N \neq 2), Q_{2}$ is the gradient $G$.

The field homogeneity is the field deviation from an ideal multipolar field. The complex magnetic field, Eq. (1), produced in a real multipolar structure can be rewritten as

$$
B(z)=C_{N}\left(\frac{z}{R_{0}}\right)^{N-1}+\sum_{n=N}^{\infty} C_{n}\left(\frac{z}{R_{0}}\right)^{n-1},
$$

where $C_{N}\left(\frac{z}{R_{0}}\right)^{N-1}$ is the main multipole and $\sum_{n \neq N}^{\infty} C_{n}\left(\frac{z}{R_{0}}\right)^{n-1}$ contains the multipole errors. The multipole errors should be reduced to come closer to an ideal structure. For a dipole, the relative field error is defined by the following expression:

$$
\frac{\Delta B}{B_{0}}=\frac{B(z)-B_{0}}{B_{0}}=\frac{1}{C_{1}} \sum_{n=2}^{\infty} C_{n}\left(\frac{z}{R_{0}}\right)^{n-1},
$$

where $B_{0}$ is the on-axis magnetic induction. For a quadrupole, the relative gradient error is defined as

$$
\frac{\Delta G}{G_{0}}=\frac{1}{C_{2}} \sum_{n>2}^{\infty}(n-1) C_{n}\left(\frac{z}{R_{0}}\right)^{n-1},
$$

where $G_{0}$ is the on-axis gradient. The homogeneity is defined as the maximum relative error in the so-called good field region (GFR).

\section{B. Other design criteria}

In many cases, additional criteria are added on top of the above magnetic requirements. They can be dedicated constraints put on the compactness or the cost, including running cost. The compactness and the magnet mass have direct consequences on the dimensioning of the magnet support system. In some applications, this can be very demanding when the available space is limited. The manufacturing cost is linked to requirements of mechanical tolerances and the complexity of the assembly. The operation cost or running cost is presently a non-negligible parameter including the electrical power consumption and the cooling power as well.

\section{HIGH GRADIENT PM QUADRUPOLE DESIGN}

The ESRF-EBS high gradient quadrupole design is based on conventional resistive technology $[13,14,16,17]$.
TABLE I. High gradient quadrupole specifications for the PM prototype described here and the EBS quadrupoles. The GFR is elliptical.

\begin{tabular}{lccc}
\hline \hline & PM prototype & EBS quads & \\
\hline Gradient & 85 & 90 & $\mathrm{~T} / \mathrm{m}$ \\
Iron length & 226 & 484 & $\mathrm{~mm}$ \\
Bore radius & 12 & 12.5 & $\mathrm{~mm}$ \\
Horizontal GFR & \pm 7 & \pm 7 & $\mathrm{~mm}$ \\
Vertical GFR & \pm 5 & \pm 5 & $\mathrm{~mm}$ \\
Vertical gap & 10 & 11 & $\mathrm{~mm}$ \\
$\Delta G / G_{0}$ & $\leq 10^{-3}$ & $\leq 10^{-3}$ & \\
\hline \hline
\end{tabular}

However, this design leads to a less compact structure compared to the PM technology. Table I gives the specifications of the PM quadrupole prototype. These specifications are close to these of the high gradient quadrupoles of the ESRF-EBS. An important aspect of these specifications is the vertical gap used for the x-ray beam ports. This constraint, specific to accelerators wherever synchrotron radiation is powerful, involves a severe truncation of the hyperbolic pole shape. The resulting gradient inhomogeneities can reach $1 \%$ or more. The specified nonsquare GFR (elliptical) is linked to requirements set by the beam dynamics: the electron beam size is not the same in the horizontal and the vertical planes.

\section{A. Magnet layout}

Iron dominated, hybrid PM technology is suitable for reaching the specifications given in Table I. The use of iron poles reduces the sensitivity of the gradient homogeneity to magnetic errors in the magnet blocks. Moreover, vertical gaps for x-ray beam ports are easy to implement on hybrid PM structures but not convenient for Halbach magnets. The PM quadrupole design studied in this paper is shown in Fig. 1. One can notice that this structure has similarities with a wiggler configuration oriented perpendicularly to the electron beam path. Simple cuboidal magnet blocks are used. The iron circuit includes four poles with an optimized shape and a return yoke. This structure is not four-way symmetric due to the arrangement of the PM blocks: it introduces octupolar terms in the multipole expansion. The reduction of these harmonics is done through an optimization of the pole shape.

\section{B. Analytical model}

The dimensions of the PM blocks and iron parts should be determined in order to reach the specified gradient. Numerical simulations using a 3D magnet model are necessary to accurately define these parameters. However an analytic study based on a 2D model is found to give initial values for these parameters. Generally speaking, 2D analytical models based on the Ampere law and flux conservation give low accuracy results due to the iron saturation and the approximations made on the 


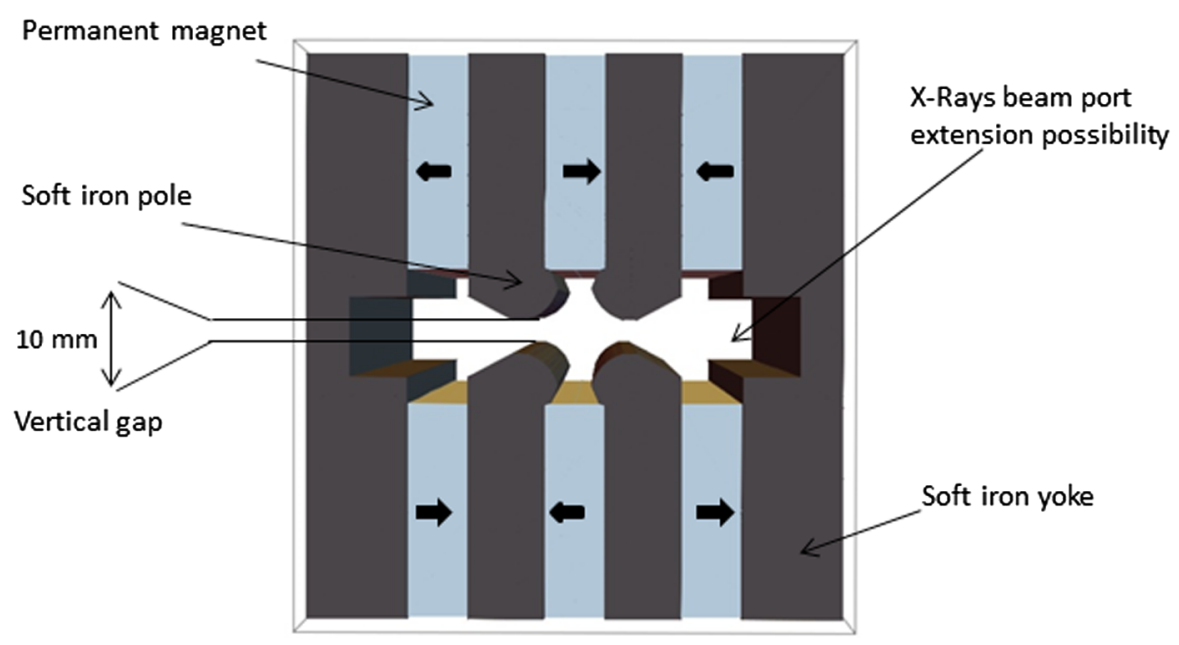

FIG. 1. H-type hybrid PM quadrupole design.

flux distribution and the fringe field. A non-negligible part of the magnetic flux may not reach the inner aperture when ferromagnetic material is close to saturation. In Ref. [15], Tosin proposed a 2D model of a hybrid PM quadrupole including the flux leakage in a bounded area. Our analytic study is based on this model. Figure 2 presents a quarter of the quadrupole. The different parameters used in the model are shown.

According to [15], the gradient is

$$
G=\frac{B_{R} h_{a}}{\frac{R_{1}^{2}}{2}-\frac{x_{1}^{2} h_{a}}{w_{a}}\left[\frac{1}{2}+\frac{x_{3}}{x_{1}-x_{3}} \ln \left(\frac{x_{3}}{x_{1}}\right)\right]},
$$

where $B_{R}$ is the remanent field of the magnet blocks. From Eq. (6), one can derive the expression of the thickness $h_{a}$ and the height $w_{a}$ of the PM blocks corresponding to the minimum PM volume per unit length $[16,17]$ :

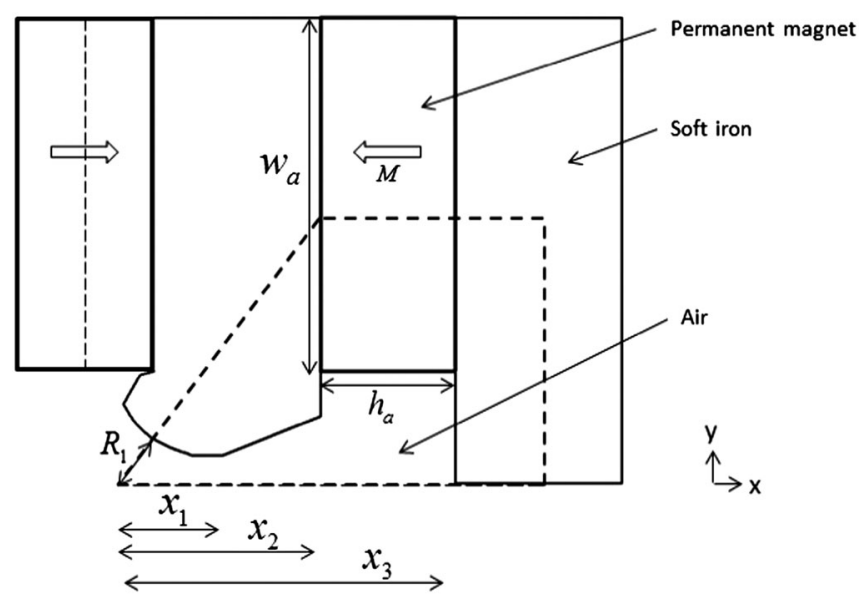

FIG. 2. Hybrid quadrupole geometrical dimensions. The contour integral for Ampere's theorem computation is shown. All PM have the same dimension for simplicity. PM can be placed on poles top to increase the gradient.

$$
\begin{gathered}
h_{a}=\frac{G R_{1}^{2}}{B_{R}} \\
w_{a}=\frac{G x_{1}^{2}}{B_{R}}\left[\frac{2 x_{3}}{x_{3}-x_{1}} \ln \left(\frac{x_{3}}{x_{1}}\right)-1\right] .
\end{gathered}
$$

From Eqs. (7) and (8), $h_{a}$ depends only on the radius $R_{1}$ and $w_{a}$ is determined by $x_{1}$ and $x_{3}$. In our application, the gradient $G$ and the inner radius $R_{1}$ are given (Table I). The vertical gap between the iron poles and the radius $R_{1}$ defines the length $x_{1}$. The length $x_{3}=x_{2}+h_{a}$ is chosen in order to avoid the saturation of the poles and to limit the flux leakage. The optimal dimensions of the PM blocks are given in Table II, assuming a $1.1 \mathrm{~T}$ remanent field in the PM blocks. This value is relatively low compared to the remanence of $\mathrm{NdFeB}$ materials, which can reach $1.42 \mathrm{~T}$ at room temperature [18]. However, high remanence grades of $\mathrm{NdFeB}$ materials are more sensitive to radiation damage than low remanence grades. (The $\mathrm{Sm}_{2} \mathrm{Co}_{17}$ material has a strong resistance to radiation damage and is particularly suitable for PM accelerator magnets. The prototype was built with $\mathrm{NdFeB}$ magnets recycled from an old undulator, due to their availability in the lab.)

The 3D simulation of the quadrupole was performed with RADIA (Fig. 3), a magnetostatic computer code developed by the ESRF ID lab and based on a volume integral method $[19,20]$. The quadrupole model is fully parametrized and includes an accurate description of the magnetic materials. The iron material is a low carbon steel AISI 1006 with $\mathrm{C}<0.06 \%$ and a high saturation magnetization. The on-axis gradient obtained with this model is

TABLE II. Optimal dimensions of the PM blocks, assuming Table I specifications, $B_{R}=1.1 \mathrm{~T}, \quad x_{1}=18 \mathrm{~mm} \quad$ and $x_{3}=50 \mathrm{~mm}$.

\begin{tabular}{llc}
\hline \hline$h_{a}$ & 11.1 & $\mathrm{~mm}$ \\
$w_{a}$ & 54.9 & $\mathrm{~mm}$ \\
\hline \hline
\end{tabular}




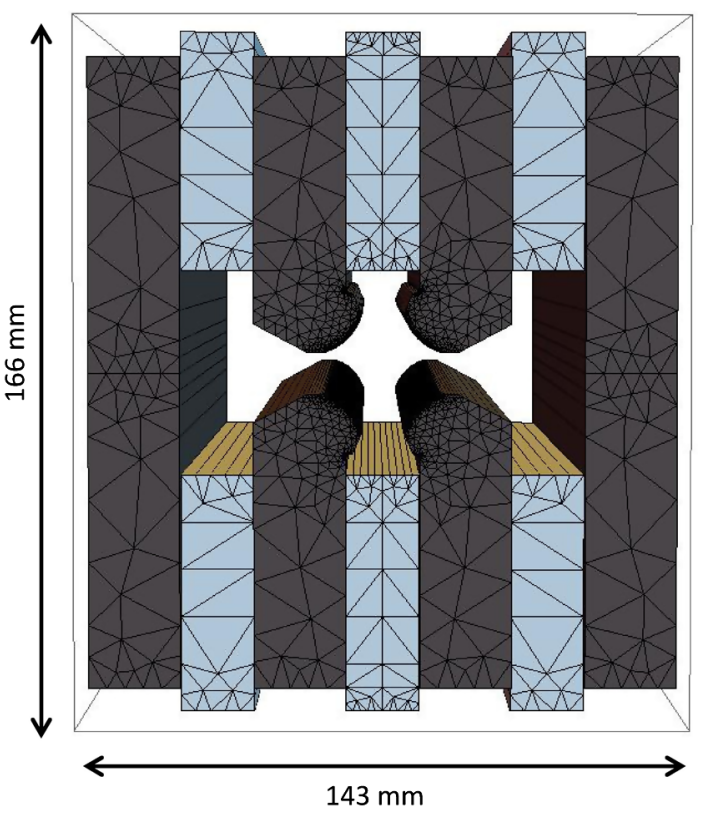

FIG. 3. 3D view of the RADIA quadrupole model including the mesh. The mesh is based on multiple polyhedrons. The model is divided into ten longitudinal slices concentrated on the edge. The pole tip has a higher mesh density because the field computation accuracy mainly depends on this area. Several simulations with different mesh refinements on the pole shape are done to check the stability of the results.

$$
G_{\text {sim }}=71.2 \mathrm{~T} / \mathrm{m}
$$

which is $16 \%$ below the gradient computed analytically. This discrepancy comes from the approximations of the 2D model: simplified flux leakage model, iron in its linear range, eightfold magnet symmetry, and perfectly rigid PM material (i.e. the magnetic susceptibility is neglected).

From the initial geometry, the sizes of the PM blocks have to be increased in order to reach the required gradient. As shown in Fig. 3, the magnet blocks are moved vertically away from the beam axis in view of reducing radiationinduced demagnetization in the PM material [21]; it also has a positive impact on the sensitivity to magnet block errors (see Sec. IV). This modification introduces a gradient reduction, which is found to decrease linearly with PM displacement (Fig. 4) in spite of pole vertical length optimization. The sensitivity of the gradient to the PM vertical displacement is approximately $1 \mathrm{~T} / \mathrm{m}$ per millimeter displacement, which leads to a $5 \mathrm{~T} / \mathrm{m}$ decrease of the gradient in the present design.

According to the above considerations, the size of the PM blocks needs to be increased to reach the specified gradient. The resulting values are

$$
\begin{aligned}
h_{a} & =18 \mathrm{~mm} \\
l_{a} & =63 \mathrm{~mm} .
\end{aligned}
$$

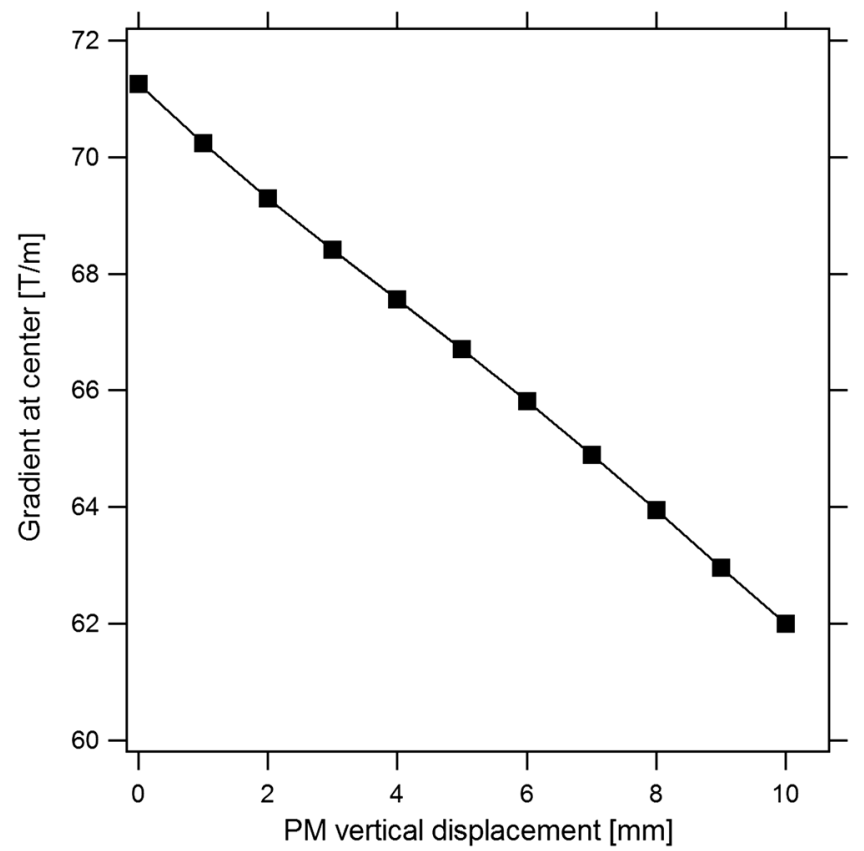

FIG. 4. Gradient reduction with PM vertical displacement.

The integrated gradients in the horizontal and vertical planes are plotted in Fig. 5. The on-axis integrated gradient of the quadrupole reaches $18.6 \mathrm{~T}$. Considering a magnetic length $L_{m} \approx L_{\text {Iron }}+2 / 3 R_{1} \approx 234 \mathrm{~mm}$, one obtains a normalized integrated gradient $\int \mathrm{Gdl} / L_{m} \approx 79.5 \mathrm{~T} / \mathrm{m}$ close to the $85 \mathrm{~T} / \mathrm{m}$ gradient specification.

\section{Pole shape optimization}

The truncation of the pole shape introduces a significant contribution from systematic harmonics of odd order $(n=6,10,14, \ldots)$ and the asymmetry of the structure also

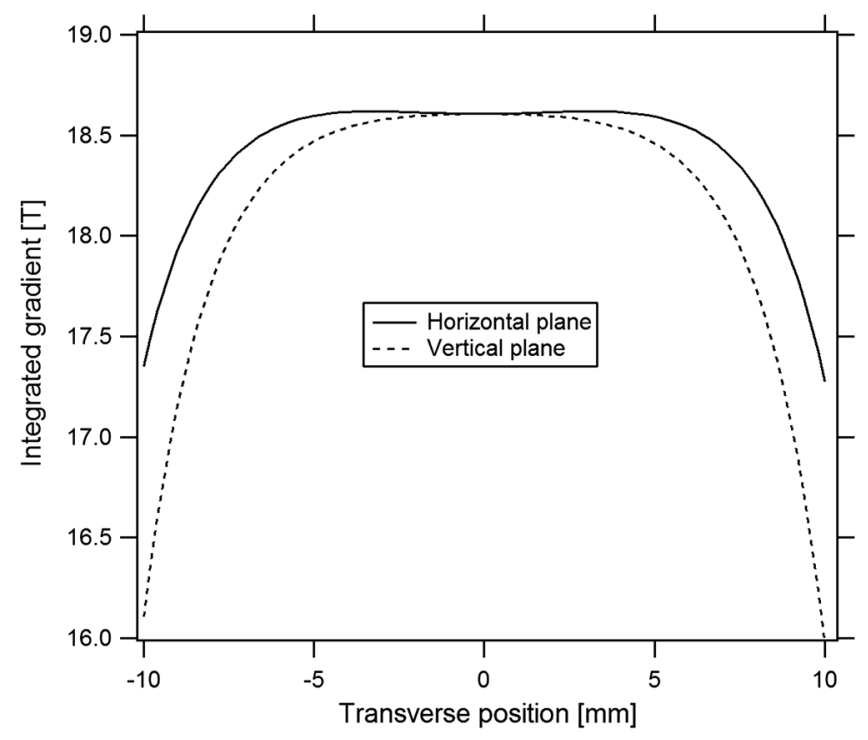

FIG. 5. Integrated gradient simulation, computed with RADIA. 
TABLE III. First normalized systematic integrated harmonics $b_{N} / b_{2}$, expressed at $7 \mathrm{~mm}$, for hyperbolic and optimized pole shapes (RADIA simulations).

\begin{tabular}{lrr}
\hline \hline$N$ & Hyperbolic & Optimized \\
\hline 4 & $2 \times 10^{-3}$ & $-5 \times 10^{-5}$ \\
6 & $-3 \times 10^{-3}$ & $-9 \times 10^{-5}$ \\
8 & $3 \times 10^{-4}$ & $-3 \times 10^{-6}$ \\
10 & $-2 \times 10^{-4}$ & $8 \times 10^{-7}$ \\
\hline \hline
\end{tabular}

introduces systematic harmonics $(n=4,8,12, \ldots)$. The first harmonics and the gradient inhomogeneities are given in Tables III and IV.

The hyperbolic profile leads to inhomogeneities 10 times higher than the $\Delta G / G_{0} \leq 10^{-3}$ specification. A modification of the pole shape is mandatory to reduce the higher harmonics and to improve the homogeneity of the gradient. We define an objective function for a minimization algorithm:

$$
\varepsilon=\sqrt{\sum_{n>2}^{\infty} b_{n}^{2}}
$$

The coordinates of the pole shape points are parametrized. An optimization algorithm is used to find the optimal position of these points, which minimize the objective function and satisfy the constraint of the minimum vertical gap between poles. This problem is complex because each point has an impact on the objective function and this impact is nonlinear with the displacement of the points. Many optimization methods can be used to solve this problem, such as determinist methods (Gauss-Newton) or random methods (Nelder-Mead simplex, genetic algorithm) presented in [22]. In order to obtain a solution in a limited number of iterations, a regularized Gauss-Newton method is chosen. This method is fully described in [23]. However a brief description is presented in the following paragraph. This minimization problem can be linearized and expressed as a least squares problem:

$$
\min _{\mathbf{X} \in \in^{\circ \mathrm{n}}}\left\|\mathbf{A}_{k} \mathbf{X}_{k}-\mathbf{b}_{k}\right\|^{2}
$$

where $k$ is the iteration number, $\mathbf{A}_{k} \in{ }^{\mathrm{om} \times \mathrm{n}}$ is the Jacobi matrix containing variations of the objective function

TABLE IV. Gradient inhomogeneities for hyperbolic and optimized pole shapes. The inhomogeneities are expressed at $7 \mathrm{~mm}$ in the horizontal plane and $5 \mathrm{~mm}$ in the vertical plane (RADIA simulations).

\begin{tabular}{lcc}
\hline \hline & Hyperbolic & Optimized \\
\hline Horizontal & $10^{-2}$ & $7 \times 10^{-4}$ \\
Vertical & $7 \times 10^{-3}$ & $5 \times 10^{-5}$ \\
\hline \hline
\end{tabular}

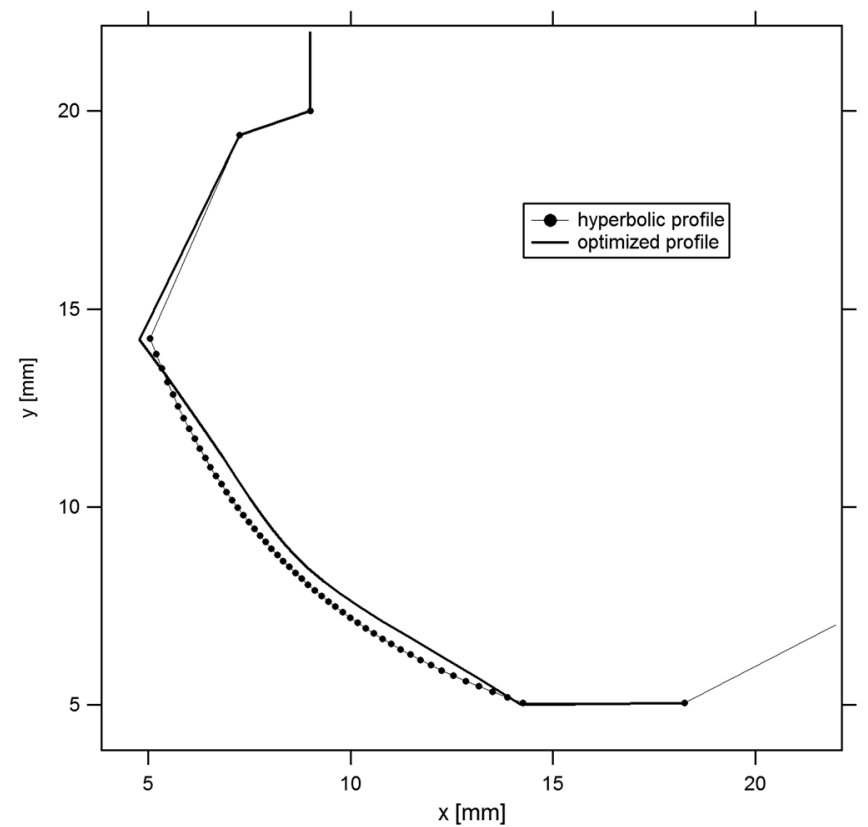

FIG. 6. Hybrid quadrupole pole shape before and after optimization.

relative to the control vector $\mathbf{X}_{k} \in{ }^{\text {on }}$ which contains pole shape points coordinates and $\mathbf{b}_{k} \in{ }^{\mathrm{om}}$ is the objective function vector containing harmonics errors and a penalty driven by the vertical gap between poles. The least square solution to Eq. (10) is

$$
\mathbf{X}_{k+1}=\mathbf{X}_{k}-\left(\mathbf{A}_{k}^{\mathrm{T}} \mathbf{A}_{k}\right)^{-1} \mathbf{A}_{k}{ }^{\mathrm{T}} \mathbf{b}_{k} .
$$

Then, a new control vector $\mathbf{X}_{k+1}$ is computed at the next iteration. The term $\left(\mathbf{A}_{k}{ }^{\mathrm{T}} \mathbf{A}_{k}\right)^{-1} \mathbf{A}_{k}{ }^{\mathrm{T}}$ is the pseudoinverse of the Jacobi matrix, which is generally ill-conditioned leading to possible divergence. Improvement of this matrix conditioning is achieved using singular value decomposition (SVD) tools. In order to reduce computation time of the Jacobi matrix $\mathbf{A}_{k}$, the control vector $\mathbf{X}_{k}$ is generated by an orthogonal polynomial, the coefficients of which are the new control variables of the pole shape. This algorithm gives a solution in less than 5 iterations.

The initial and the optimized pole are presented Fig. 6 . The optimized pole shape is close to the initial hyperbola shape, partly due to the SVD of the Jacobi matrix, which limits the solution norm. The effect of this optimization on the field quality is given in Tables III and IV. The homogeneity specifications are satisfied in both planes. The amplitudes of all harmonics are reduced.

\section{IMPACT OF MAGNETIC AND MECHANICAL ERRORS}

The dependence of the field quality on different types of errors is an essential subject requiring a dedicated study. Magnetic errors are imperfections of PM characteristics 


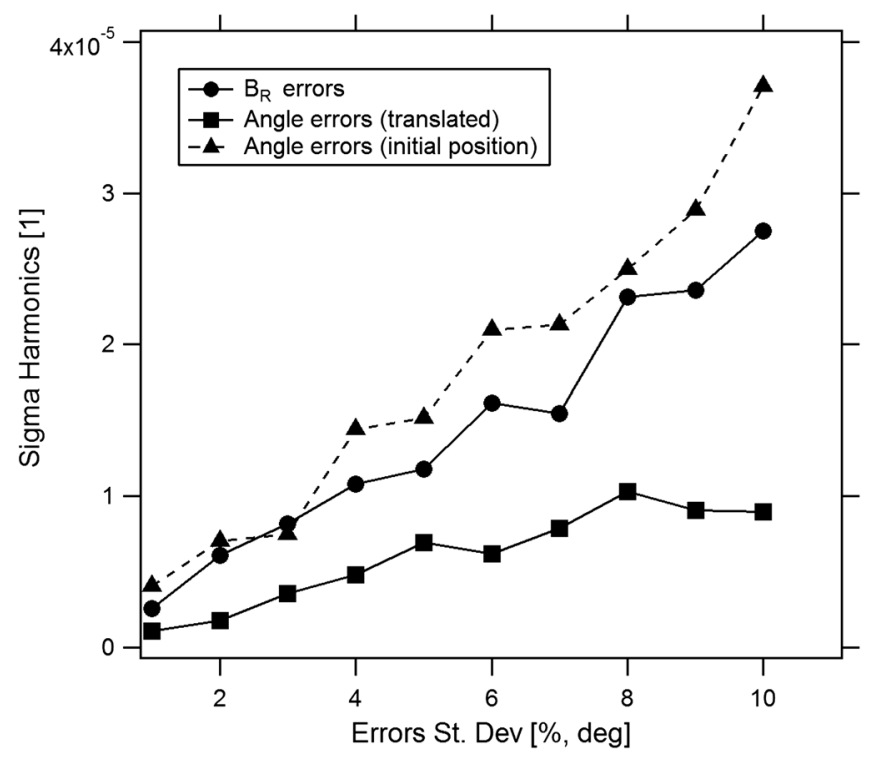

FIG. 7. PM sensitivity to magnetic errors. Errors are in percent and angle errors are in degrees. Initial position: PM blocks without vertical translation; translated: PM blocks with $5 \mathrm{~mm}$ translation. Six blocks with independent magnetic errors are installed in each slot.

such as the fluctuations of remanent induction and magnetization direction from the ideal characteristics. Mechanicals errors are linked to assembly imperfections and also pole shape machining errors. It is assumed that these errors are random, therefore favoring a statistical study approach.

Errors with Gaussian distribution were introduced in the 3D model. The field errors have been estimated by setting nonzero standard deviation for each type of error, then computing the field harmonic distortion $\sigma_{\text {Harmonic }}{ }^{2}=\left({\sigma_{a_{3}}}^{2}+\cdots+{\sigma_{a_{N}}}^{2}+{\sigma_{b_{3}}}^{2}+\cdots+{\sigma_{b_{N}}}^{2}\right) / b_{2}{ }^{2} \approx$ $2\left({\sigma_{b_{3}}}^{2}+\cdots+{\sigma_{b_{N}}}^{2}\right) / b_{2}{ }^{2}$ from 40 samples. This procedure was iterated with different standard deviations and for all of the errors considered. The results of these simulations are shown in Figs. 7 and 8. The simulations of angular errors in PM blocks are done without vertical translation of the PMs and with $5 \mathrm{~mm}$ vertical translation (angular errors are deviations from the specified direction of magnetization). The assembly errors are assumed to be positioning errors of the poles in the horizontal and vertical planes.

The simulations show that the mechanical errors have a higher impact on the field quality than the magnetic errors of the PM blocks. Assembly and pole shape errors (deviation from the optimized shape, modeled by random displacement of the profile points) have roughly the same impact on field quality. Vertical translations of PMs away from the midplane have the beneficial effect of reducing sensitivity to PM magnetization direction error.

The magnet sensitivity to the different classes of error can be expressed as

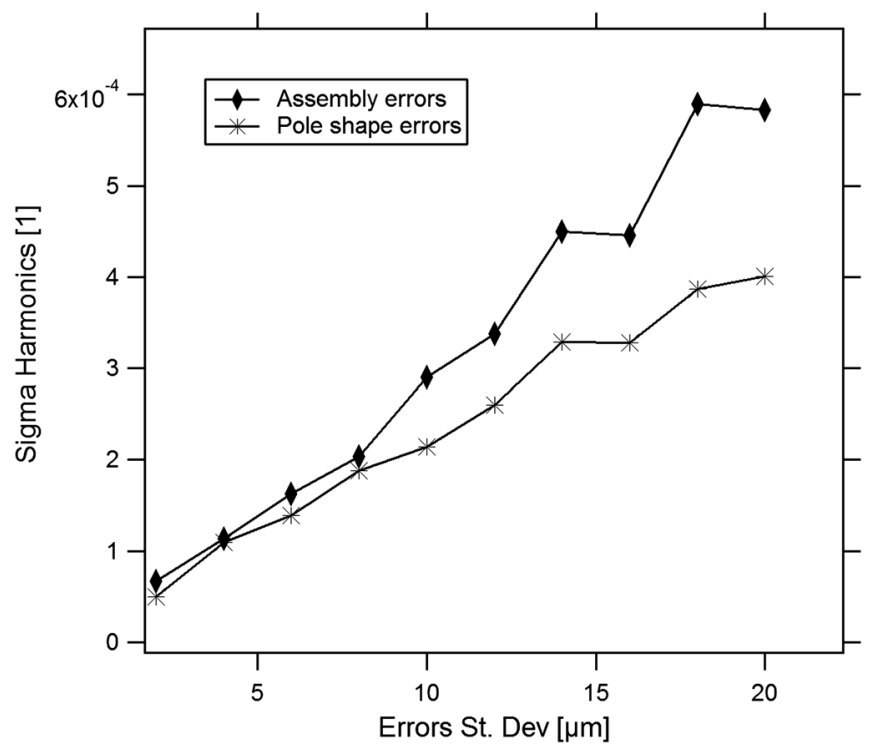

FIG. 8. Simulations of magnet sensitivity to mechanical errors.

$\sigma_{\text {harmonics }}$
$\quad=\sqrt{\left(k_{\mathrm{B}_{\mathrm{R}}} \sigma_{\mathrm{B}_{\mathrm{R}}}\right)^{2}+\left(k_{\theta} \sigma_{\theta}\right)^{2}+\left(k_{\text {Assy }} \sigma_{\text {Assy }}\right)^{2}+\left(k_{\text {shape }} \sigma_{\text {shape }}\right)^{2}}$,

where $k_{\mathrm{B}_{\mathrm{R}}}, k_{\theta}, k_{\mathrm{Assy}}, k_{\text {shape }}$ are the sensitivity of the harmonics to the corresponding errors and $\sigma_{\mathrm{B}_{\mathrm{R}}}, \sigma_{\theta}$, $\sigma_{\text {Assy }}, \sigma_{\text {shape }}$ are associated standard deviations. The sensitivities of the harmonics for each type of error are given in Table V.

As shown in Table V, the field quality of the PM quadrupole mainly depends on the mechanical errors. The contribution of the magnetic errors is about 2 orders of magnitude smaller than that of the mechanical errors if one considers mechanical errors in the $10 \mu \mathrm{m}$ range, angular errors around $1^{\circ}$ and magnetization errors around $1 \%$. This result is interesting because one can relax the magnetic tolerances on the PM blocks and reduce the resulting procurement cost.

Assuming a Gaussian distribution for the harmonic errors, $3 \sigma_{\text {harmonics }} \leq 10^{-3}$ (i.e. $0.1 \%$ of the magnets are expected to be out of the tolerances) and identical contributions from the assembly and shape errors, the standard deviations for the mechanical errors should comply with

TABLE V. Harmonic sensitivity coefficients (PM blocks translated vertically), assuming 6 PM blocks in each slot.

\begin{tabular}{lccc}
\hline \hline Errors & Coefficient & Value & Unit \\
\hline$B_{R}$ & $k_{\mathrm{B}_{\mathrm{R}}}$ & $2.7 \times 10^{-6}$ & $1 / \%$ \\
Angle & $k_{\theta}$ & $1 \times 10^{-6}$ & $1 /{ }^{\circ}$ \\
Assembly & $k_{\text {Assy }}$ & $3.1 \times 10^{-5}$ & $1 / \mu \mathrm{m}$ \\
Poles shape & $k_{\text {shape }}$ & $2 \times 10^{-5}$ & $1 / \mu \mathrm{m}$ \\
\hline \hline
\end{tabular}



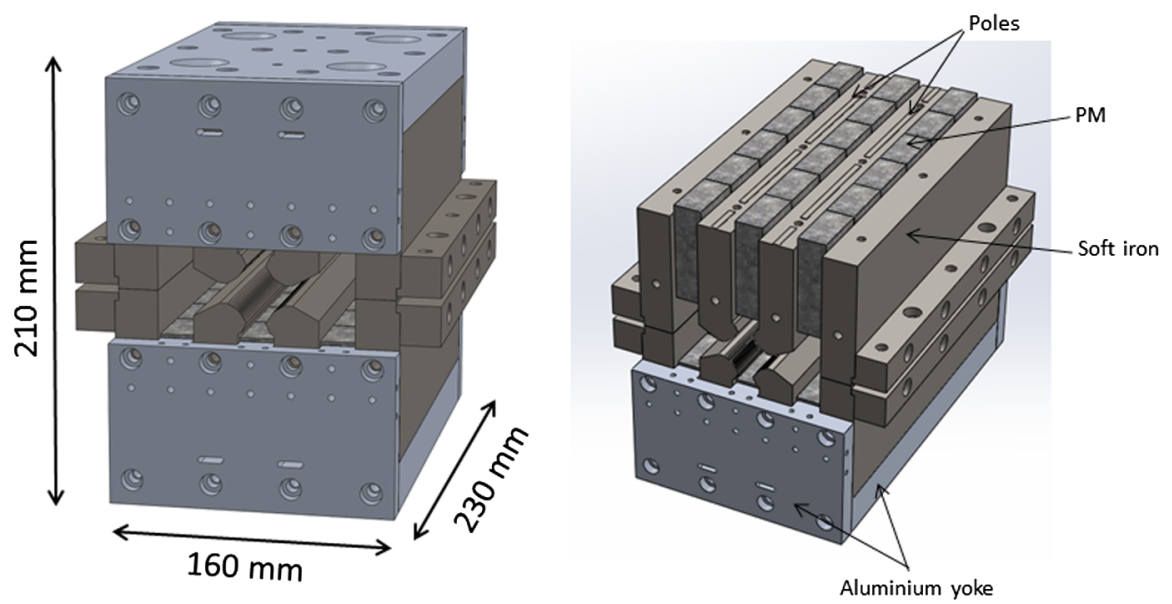

FIG. 9. Hybrid PM prototype CAD model. Dimensions of the prototype are indicated (left). The upper mechanical parts of the quadrupole are removed to show the PM arrangement in slots (right). The total mass of this prototype is $45 \mathrm{~kg}$ and the weight of PM material is $12 \mathrm{~kg}$.

$$
\begin{gathered}
\sigma_{\text {Assy }}=\sigma_{\text {harmonics }} / k_{\text {Assy }} \leq 8 \mu \mathrm{m} \\
\sigma_{\text {shape }}=\sigma_{\text {harmonics }} / k_{\text {Shape }} \leq 12 \mu \mathrm{m}
\end{gathered}
$$

This gives the global tolerance for the pole shape:

$$
\begin{aligned}
\text { tol } & = \pm \sqrt{3} \sigma_{\text {Shape }} \\
& \approx \pm 21 \mu \mathrm{m}
\end{aligned}
$$

and for the assembly tolerance,

$$
\begin{aligned}
\text { tol } & = \pm \sqrt{3} \sigma_{\text {Assy }} \\
& \approx \pm 14 \mu \mathrm{m}
\end{aligned}
$$

assuming a uniform distribution of the mechanical errors. These tolerances are tight and are not trivial to obtain. In particular, one can expect positioning errors larger than indicated above. Loosening the magnetic tolerances to $2 \sigma_{\text {harmonics }} \leq 10^{-3}$ (5\% of the magnets out of the tolerances) would lead to a $\pm 35 \mu \mathrm{m}$ global tolerance.

\section{PROTOTYPE AND MEASUREMENT}

\section{A. Fabrication of the prototype}

Compared to a conventional resistive structure realized with fewer parts and limited degrees of freedom, this design involves several parts, which leads to tolerance stack-up. An aluminum yoke ensures the positioning of the poles (Fig. 9). The yoke is composed of three parts, one of which is located on the top of poles in order to set the vertical position and the others located on the front and back of the poles to ensure their transverse positions. The vertical positions of the poles can be adjusted with spacers. The force applied on each magnetic element (PM and poles) was computed. These computations show that PM blocks are pushed upward toward the top of the poles so they are maintained in contact with the top yoke. Jigs parts have been used to guide PM in their location during assembly.

The poles and iron yoke are magnetized by $36 \mathrm{NdFeB}$ PM blocks distributed in six slots. The magnetic moments $m$ of the PM blocks and their axes were measured with a three axis sensor fluxgate placed at a large distance $d$ from the blocks such that the measured field is $B=\mu_{0} m /\left(2 \pi d^{3}\right)$, with $d=700 \mathrm{~mm}$. Four measurements per block are necessary to determine each field component and to eliminate the ambient field. The remanence of the magnet blocks was derived from the average magnetization, assuming a magnetic permeability of 1.05 for the PM material. Figure 10 shows the dispersion of remanence and magnetization angle errors for the measured PM blocks.

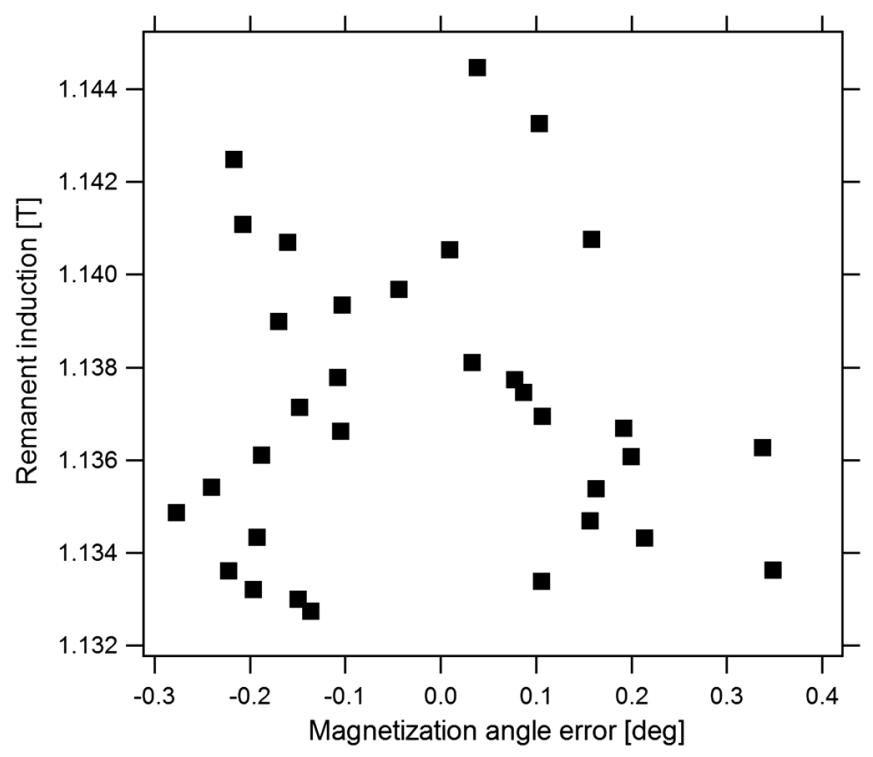

FIG. 10. Characteristics of the $36 \mathrm{NdFeB}$ PMs used in the prototype. The average remanent field is $\mathrm{BR}=1.137 \mathrm{~T}$. These $\mathrm{PM}$ are recycled from an old undulator. 


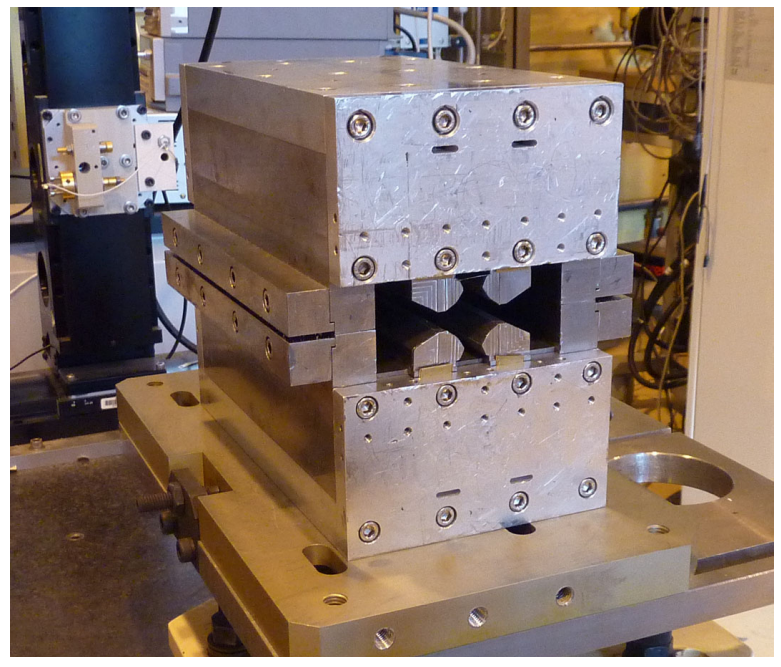

FIG. 11. Quadrupole prototype installed on a stretched wire measurement bench.

The PM blocks have been sorted according to these measurements before their installation in the six slots of the prototype.

\section{B. Magnetic measurement}

The magnetic measurements of the quadrupole prototype were performed with a stretched wire measurement bench [24] (Fig. 11). The gradient of the prototype reaches $82 \mathrm{~T} / \mathrm{m}$, which is in agreement with the simulation results. The higher order multipoles are given in Table VI. The transverse and vertical homogeneities of the gradient have been computed and compared to the ideal case (Fig. 12).

The homogeneity of the integrated gradient in the vertical plane is far from expectation. Besides the displacement of the poles in the vertical direction, additional degrees of freedom would have been useful but difficult to implement. For example, one can mention the positioning of the poles in the horizontal direction.

The integrated gradient homogeneity has been corrected using magnetic shims. The shims have the same contours as the poles (Fig. 13) and a varying thickness controlled with six machined slots. The depth of each slot is determined in order to correct the integrated gradient errors and restore a larger transverse homogeneity. The approach is similar to

TABLE VI. Higher order integrated multipoles of the quadrupole prototype, measured at $10 \mathrm{~mm}$ and expressed at $7 \mathrm{~mm}$ radius. The other multipoles are below $10^{-5}$.

\begin{tabular}{lrr}
\hline \hline $\mathrm{n}$ & $b_{n} / b_{2}$ (normal) & $a_{n} / b_{2}$ (skew) \\
\hline 3 & $-1.3 \times 10^{-4}$ & $3.8 \times 10^{-4}$ \\
4 & $3 \times 10^{-4}$ & $0.5 \times 10^{-4}$ \\
6 & $-8.4 \times 10^{-4}$ & $1.9 \times 10^{-4}$ \\
10 & $-7 \times 10^{-4}$ & $-0.7 \times 10^{-4}$ \\
12 & $-2.5 \times 10^{-4}$ & $1.1 \times 10^{-4}$ \\
\hline \hline
\end{tabular}
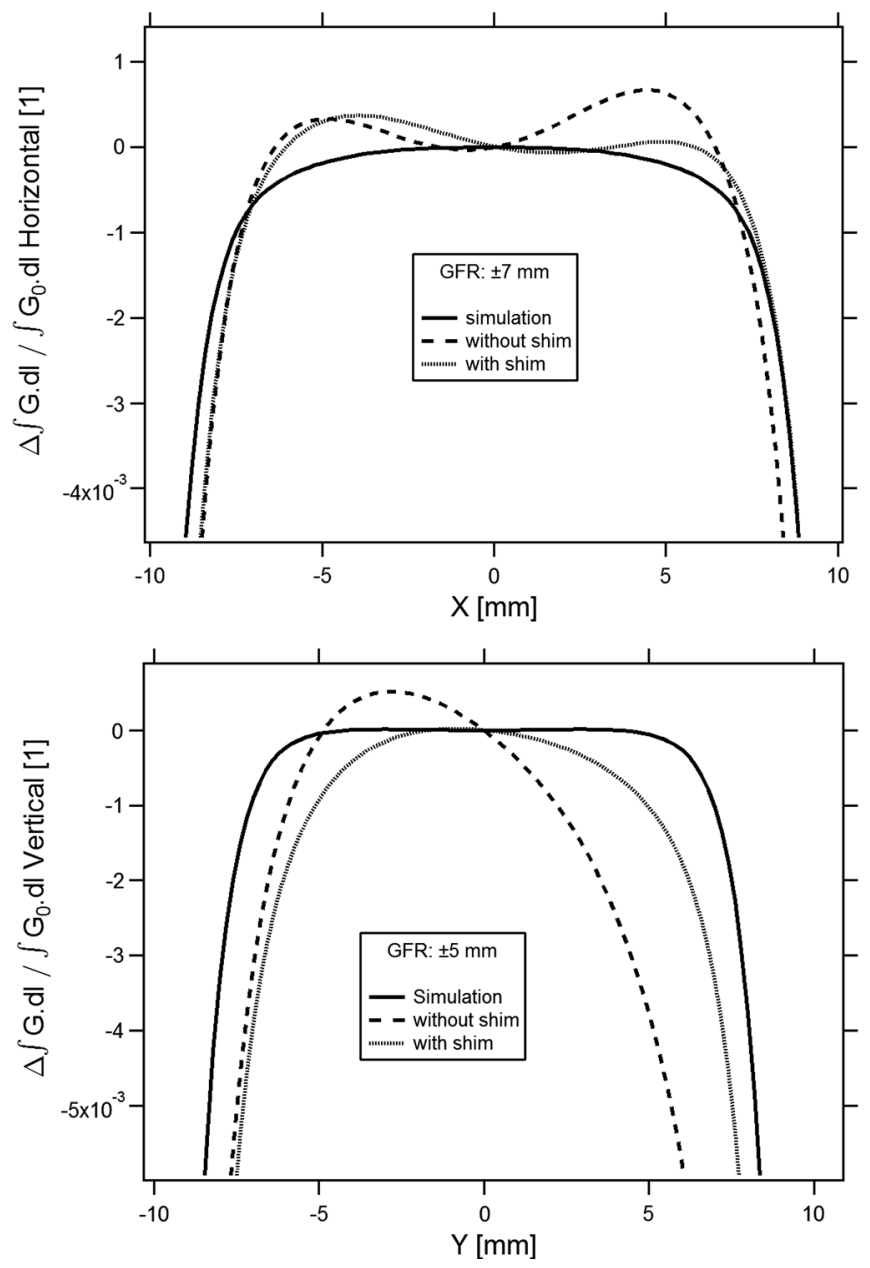

FIG. 12. Homogeneity of the integrated gradient along the horizontal and vertical axes. Simulation and measurement results are plotted. Results after correction shim implementation are shown.

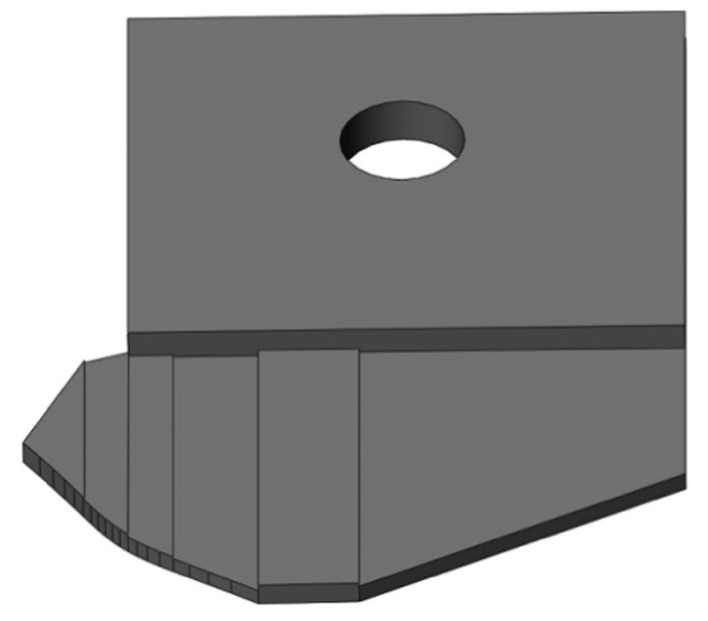

FIG. 13. Correction shim profile. The machined shape is composed of six slots with different thickness. The shim material is steel AISI 1006. 
the technique used for the shimming of undulators [25]. The computation method is based on a linear system solved by a direct matrix inversion. This correction method leads to good results as shown in Fig. 12.

\section{CONCLUSION}

The magnets to be used in new storage ring-based light sources are clearly evolving towards a much smaller bore radius. This is the primary parameter enabling higher performance for multipole magnets. As a consequence, PM technology has good potential when the compactness and running costs of such devices are important figures of merit. A simple PM-based high gradient quadrupole prototype has been built and magnetically characterized. The proposed structure has a low sensitivity to the magnetic errors in the magnet blocks due to the use of soft iron material for the poles. The field quality of the device depends mostly on mechanical errors. It can be improved with a higher performing poles positioning than that used for the prototype. The use of special iron-type shims leads to a net improvement in field quality. A gradient homogeneity better than $10^{-3}$ was obtained after installation of these shims.

[1] D. Einfeld, Multibend achromat lattices for storage ring light sources, Synchrotron Radiat. News 27, 4 (2014).

[2] L. Farvacque et al., A low emittance lattice for the ESRF, in Proceedings of the 4th International Particle Accelerator Conference, IPAC-2013, Shanghai, China, 2013 (JACoW, Shanghai, China, 2013), p. 79.

[3] K. Halbach, Nucl. Instrum. Methods Phys. Res., Sect. A 187, 109 (1981).

[4] G. W. Foster, in Proceedings of the 11th European Particle Accelerator Conference, Genoa, 2008 (EPS-AG, Genoa, Italy, 2008), p. 189.

[5] Y. Iwashita et al., Super strong adjustable permanent magnet quadrupole for the final focus in a linear collider, in Proceedings of the 10th European Particle Accelerator Conference, Edinburgh, Scotland, 2006 (EPS-AG, Edinburgh, Scotland, 2006).

[6] M. Modena et al., Design, assembly and first measurements of a short model for CLIC final focus hybrid quadrupole, in Proceedings of the 3rd International Particle Accelerator Conference, New Orleans, LA, 2012 (IEEE, Piscataway, NJ, 2012).

[7] Shepherd et al., Construction and measurement of novel adjustable permanent magnet quadrupole for CLIC, in
Proceedings of the 3rd International Particle Accelerator Conference, New Orleans, LA, 2012 (Ref. [7]).

[8] J. K. Lim et al., Adjustable short focal length permanent magnet quadrupole based electron beam final focus system, Phys. Rev. ST Accel. Beams (2005).

[9] J. Chavanne et al., Magnet for a new low emittance storage ring at ESRF, IMMW18 (Brookhaven, 2013).

[10] ESRF staff, ESRF upgrade program phase II technical design study, 2014, http://www.esrf.eu.

[11] G. Le Bec et al., Compact magnetic elements using permanent magnets, Japanese-French Symposium on Advanced Compact Free-Electron Lasers (Tokyo, 2014).

[12] O. Chubar, P. Elleaume, and J. Chavanne, J. Synchrotron Radiat. 5, 481 (1998).

[13] G. Le Bec et al., Magnets for the ESRF diffraction limited light source project, IEEE TASC 26 (2016).

[14] G. Le Bec, J. Chavanne, C. Benabderrahmane, L. Farvacque, L. Goirand, S. Liuzzo, P. Raimondi, and F. Villar, High gradient quadrupoles for low emittance storage rings, Phys. Rev. Accel. Beams 19, 052401 (2016).

[15] G. Tosin, P. P. Sanchez, J. F. Citadini, and C. C. Vergasta, Super hybrid quadrupoles, Nucl. Instrum. Methods Phys. Res., Sect. A 674, 67 (2012).

[16] G. Le Bec et al., Magnet designs for the ESRF-SR2, Workshop on Special Compact and Low Consumption Magnet Designs (CERN, Geneva, 2014).

[17] G. Le Bec and J. Chavanne, High gradient permanent magnet technology for ultra-high brightness rings, in Proceedings NAPAC2016, THA2IOO2 (Chicago, 2016).

[18] J. Chavanne et al., Prospects for the use of permanent magnets in future accelerator facilities, IPAC2014.

[19] P. Elleaume et al., Computing 3D magnetic fields from insertion devices, in Proceedings of the Particle Accelerator Conference, Vancouver, BC, Canada, 1997 (IEEE, New York, 1997), pp. 3509-3511.

[20] O. Chubar, P. Elleaume, and J. Chavanne, A threedimensional magnetostatics computer code for insertion devices, J. Synchrotron Radiat. 5, 481 (1998).

[21] T. Bizen, Y. Asano, T. Hara, X. Marechal, T. Seike, T. Tanaka, H.S Lee, D.E Kim, C.W Chung, and H. Kitamura, Nucl. Instrum. Methods Phys. Res., Sect. A 515, 850 (2003).

[22] S. Russenschuck, Field Computation for Accelerator Magnets (Wiley-VCH, New York, 2010).

[23] G. Le Bec et al., Shape optimization for the ESRF II magnets, IPAC14 (2014).

[24] G. Le bec et al., Stretched wire measurement of multipole accelerator magnets, Phys. Rev. ST Accel. Beams (2012).

[25] J. Chavanne, C. Benabderrahmane, G. Le Bec, and C. Penel, Recent developments in insertion devices at the ESRF: Working toward diffraction-limited storage rings, Synchrotron Radiat. News 28, 15 (2015). 